\title{
Avaliação da qualidade da água dos reservatórios localizados nas bacias hidrográficas dos rios Piauí - Real, utilizando o índice de qualidade da água (IQA)
}

Evaluation of the water quality of the reservoirs located in the Piauí - Real river basins, using the water quality index (IQA)

\author{
R. S. Lima ${ }^{1 *}$; J. P. H. Alves ${ }^{2}$ \\ ${ }^{1}$ Recursos Pesqueiros e Aquicultura, Instituto Federal de Sergipe, 49200-000, Estância-Sergipe, Brasil \\ ${ }_{2}^{2}$ Programa de Pós-Graduação em Recursos Hídricos, Universidade Federal de Sergipe, 49100-000, São Cristóvão- \\ Sergipe, Brasil
}

*robpesca@hotmail.com

(Recebido em 28 de maio de 2017; aceito em 28 de setembro de 2017)

\begin{abstract}
Nesse trabalho o IQA multiplicativo, desenvolvido pela NSF (National Sanitation Foundation) e adaptado no Brasil pela Companhia de Tecnologia de Saneamento Ambiental de São Paulo (CETESB), foi usado para avaliar a qualidade da água dos reservatórios Dionísio Machado (DM), Taboca (TB), Amargosa (AG) e Jabiberi (JB), situados na região Centro Sul do Estado de Sergipe. Os reservatórios DM e TB estão inseridos na bacia hidrográfica do rio Piauí, e o AG e JB na bacia do rio Real. As amostras de água de superfície foram coletadas no período de junho/2013 a agosto/2014, compreendendo dois períodos chuvosos e um período seco. Em cada amostra foram determinados os seguintes parâmetros: $\mathrm{pH}$, turbidez (TUR), oxigênio dissolvido $(\mathrm{OD})$, demanda bioquímica de oxigênio $\left(\mathrm{DBO}_{5}\right)$, sólidos totais dissolvidos (STD), fósforo total $\left(\mathrm{P}_{\mathrm{t}}\right)$, nitrato $\left(\mathrm{NO}_{3}\right)$, coliformes termotolerantes $(\mathrm{COL})$ e temperatura da água (T, medida no campo). A Análise de Componentes Principais (ACP) foi utilizada como ferramenta auxiliar para resolver a limitação do IQA, em relação aos valores de STD superiores a $500 \mathrm{mg} \mathrm{L}^{-1}$. A água do reservatório AG foi classificada como "Ruim" e a do reservatório JB como "Boa". Para os reservatórios TB e DM a qualidade da água variou de "Aceitável" para "Boa" em função do período de amostragem. Os parâmetros que mais impactaram sobre a qualidade da água foram $\mathrm{DBO}_{5}, \mathrm{OD}, \mathrm{COL}$ e STD, e devem estar associados, principalmente, ao aporte de despejos sanitários e a processos de salinização.
\end{abstract}

Palavras-chave: IQA, Análise de Componentes Principais, Reservatório.

In this work the multiplicative IQA, developed by NSF (National Sanitation Foundation) and adapted in Brazil by the São Paulo Environmental Sanitation Technology Company (CETESB), was used to evaluate the water quality of the reservoirs Dionísio Machado (DM), Taboca (TB), Amargosa (AG) and Jabiberi (JB), located in the South Central region of the State of Sergipe. The DM and TB reservoirs are located in the Piauí river basin, and the AG and JB in the Real river basin. Surface water samples were collected from June/2013 to August/2014, comprising two rainy periods and one dry period. In each sample the following parameters were determined: $\mathrm{pH}$, turbidity (TUR), dissolved oxygen (OD), biochemical oxygen demand (BOD5), total dissolved solids (STD), total phosphorus (Pt), nitrate (NO3), thermotolerant coliforms (COL) and water temperature (T, measured in the field). Principal Component Analysis (PCA) was used with an auxiliary tool to solve the limitation of the IQA, in relation to the STD values above $500 \mathrm{mg} \mathrm{L}^{-1}$. The water in the AG reservoir was classified as "Bad" and the water in the JB reservoir was "Good". For the TB and DM reservoirs the water quality ranged from "Acceptable" to "Good" depending on the sampling period. The parameters that most affected the water quality were DBO5, OD, COL and STD, and should be associated mainly with the contribution of sanitary waste and salinization processes.

Keywords: IQA, Principal Component Analysis, Reservoir.

\section{INTRODUÇÃO}

A demanda humana por água doce está próxima e em alguns lugares já excede a disponibilidade existente, por isso, a escassez de água tornou-se o maior desafio a ser enfrentado pela gestão dos recursos hídricos [7]. Convencionalmente, para atender a escassez hídrica são utilizadas águas subterrâneas profundas e o armazenamento de águas superficiais em reservatórios. Embora os $109918-1$ 
reservatórios sejam uma fonte confiável de água para atender às demandas, eles têm efeitos transformadores nos rios, incluindo a fragmentação, mudança do regime fluvial, aumento da perda por evaporação, maior tempo de permanência (residência) da água, além de consequências que afetam as comunidades aquáticas [7]. Essa mudança causa impactos ambientais, a montante e a jusante do barramento, alterando as características físicas, químicas, e biológicas da água [5, 26].

A qualidade das águas dos corpos d'águas, sobretudo, as águas de reservatórios tem sido objeto de estudo para vários pesquisadores, principalmente, quando se discute os seus usos múltiplos. Variações associadas a fatores climáticos, intervenção humana, usos da terra e aportes antropogênicos têm afetado significativamente a qualidade das águas [7].

No semiárido brasileiro a aridez e a distribuição irregular da precipitação pluviométrica no tempo e no espaço levaram à prática da construção de reservatórios de captação e de armazenamento das águas superficiais [10]. As águas represadas estão sujeitas à deterioração de sua qualidade devido à alta taxa de evaporação e aos aportes de nutrientes oriundos da agricultura e esgotos domésticos [18].

Durante as últimas décadas, várias ferramentas têm sido propostas para avaliação da qualidade da água, e uma das mais usadas são os Índices de Qualidade da Água (IQA). Os IQAs são ferramentas práticas de baixo custo, e por isso, têm sido utilizadas na avaliação da qualidade da água, em países desenvolvidos ou em desenvolvimento [1]. São indicadores compostos, da qualidade da água que juntam as informações dos diferentes parâmetros de qualidade da água em um único valor global, que pode ser rapidamente e facilmente comunicado ao público-alvo (gestores, usuários e publico em geral). Os IQA apresentam a vantagem de diminuir o número de parâmetros associados que precisam ser interpretados para determinação da qualidade da água; permitem comparar estados de diferentes corpos de água, no intervalo espaço - tempo e são úteis para o planejamento de ações de gestão e aplicação de recursos financeiros $[6,12,13,20)$.

Existem disponiveis na literatura vários IQAs [12], e muitos deles foram desenvolvidos para sistemas de água doce [19], contudo têm sido considerados adequados também para utilização em lagos [21], fontes de água potável em geral [14] e águas usadas na agrícultura [11].

Nesse trabalho, o IQA foi utilizado como ferramenta para avaliar a qualidade da água, dos reservatórios Dionísio Machado, Taboca, Amargosa e Jabiberi, situados na região Centro Sul do Estado de Sergipe. Os resultados do IQA foram comparados com o agrupamento dos reservatórios, em função da qualidade da água, obtido usando a análise estatística de Componentes Principais.

\section{MATERIAL E MÉTODOS}

A área de estudo compreende quatro reservatórios localizados na região Centro Sul do estado de Sergipe. Os reservatórios Dionísio Machado (DM) e Taboca (TB) estão inseridos na bacia hidrográfica do rio Piaú, o Amargosa (AG) e o Jabiberi (JB) na bacia do rio Real $[15,16,17]$. A Figura 1 mostra a localização dos reservatórios. A Tabela 1 mostra as principais características de cada reservatório, tais como, localização, ano de construção, volume de armazenamento, material de construção e principais usos da água.

Tabela 1: Principais características dos reservatórios

\begin{tabular}{|c|c|c|c|c|}
\hline \multirow{2}{*}{ Características } & \multicolumn{4}{|c|}{ Reservatórios } \\
\hline & DM & TB & JB & AG \\
\hline Cidade/UF & Lagarto/SE & Simão Dias/Se & Tobias Barreto/SE & $\begin{array}{c}\text { Poço } \\
\text { Verde/SE }\end{array}$ \\
\hline Localização & $\begin{array}{c}\text { Confluência (rios } \\
\text { Piauí e Jacaré) }\end{array}$ & $\begin{array}{c}\text { Barramento do rio } \\
\text { Taboca }\end{array}$ & $\begin{array}{c}\text { Barramento do rio } \\
\text { Jabiberi }\end{array}$ & $\begin{array}{c}\text { Reservatório } \\
\text { escavado }\end{array}$ \\
\hline Construção & $1985-1987$ & DNOCS - 1914 & $1986-1987$ & 1985 \\
\hline Volume $\mathbf{m}^{3}$ & 15.000 .000 & 115.000 & 4.300 .000 & 4.300 .000 \\
\hline Principais usos & $\begin{array}{l}\text { Humano e hidro } \\
\text { agrícola }\end{array}$ & $\begin{array}{c}\text { Humano e } \\
\text { irrigação }\end{array}$ & $\begin{array}{c}\text { Humano e hidro } \\
\text { agrícola }\end{array}$ & $\begin{array}{c}\text { Atualmente } \\
\text { sem uso }\end{array}$ \\
\hline
\end{tabular}




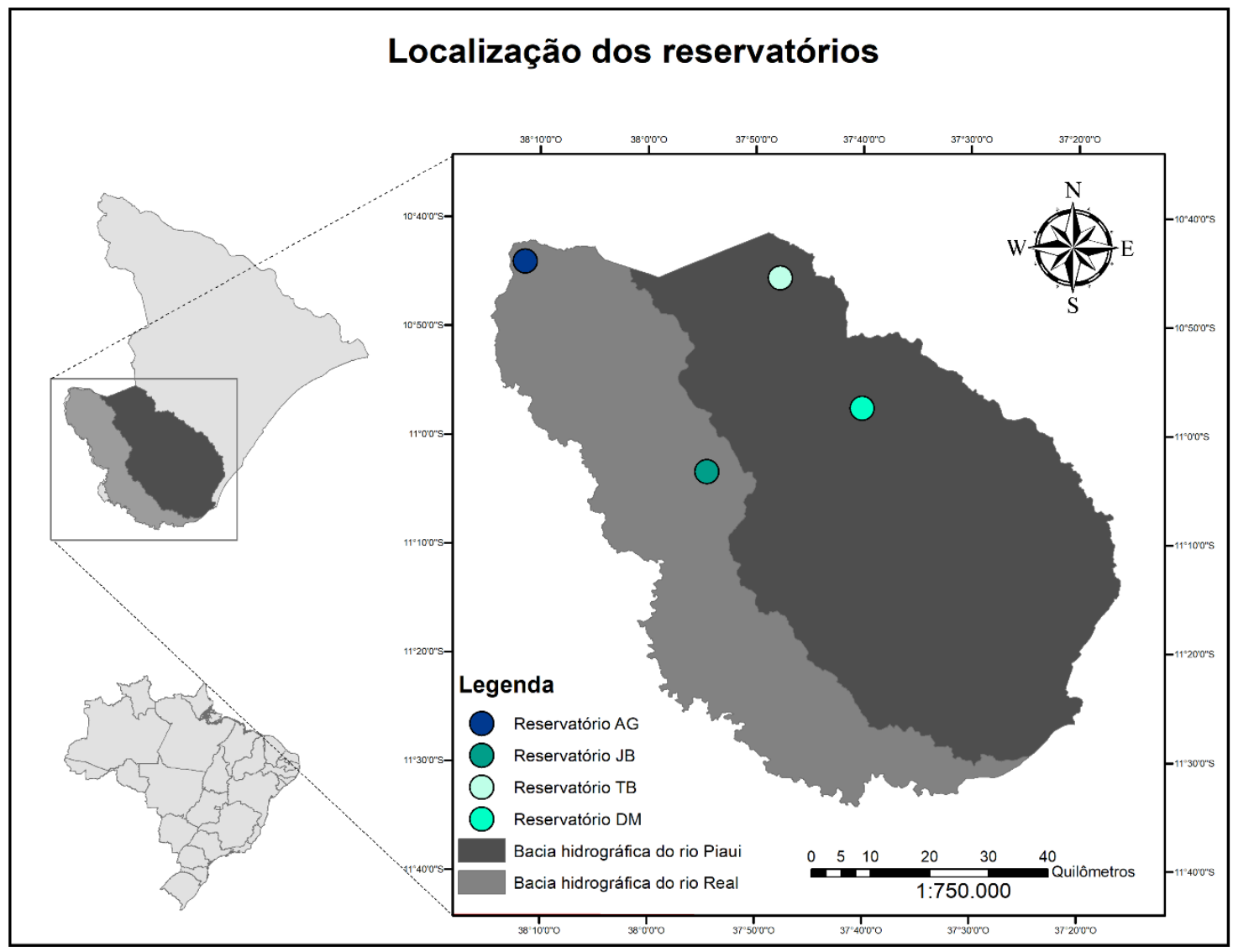

Figura 1. Localização dos reservatórios. Fonte: elaborado pelo autor

\subsection{Características gerais dos reservatórios:}

-Reservatório Dionísio Machado (DM)

O reservatório Dionísio Machado está localizado no município de Lagarto, na confluência entre os rios Piauí e Jacaré. A barragem teve as suas obras iniciadas em 1985 e foram concluídas em 1987. Este reservatório tem um volume de $15.000 .000 \mathrm{~m}^{3}$ e como principais usos, abastecimento humano e hidro agrícola. Responde por $80 \%$ do abastecimento de água da cidade de Lagarto [8].

De acordo com a classificação climática de Koppen, o clima da região é do tipo As (Clima tropical com chuvas no inverno), com precipitação média de $1.020,6 \mathrm{~mm} / \mathrm{ano}^{-1} \mathrm{e}$ as temperaturas médias do ar dos meses mais frios ocorrem em julho e agosto, com valores em trono de $22,5^{\circ} \mathrm{C}$, e o mês mais quente é janeiro, com média de $26,2^{\circ} \mathrm{C}$. As temperaturas médias mínimas mensais estão compreendidas entre 18 e $22^{\circ} \mathrm{C}$ e as temperaturas médias máximas mensais estão entre 26 e $32^{\circ} \mathrm{C}[25]$.

Os solos prevalecentes nessa região são classificados como Podzólicos Vermelho Amarelo, Plintossolos e Planossolos Solódicos [25]. Os solos do tipo Planossolos Solódicos, por serem de textura arenosa, são de baixa fertilidade e, por possuir elevada acidez, dificultam por muito tempo os cultivos agrícolas, de forma que são necessários aditivos para o cultivo [23].

-Reservatório Taboca (TB)

O reservatório Taboca foi construído em 1914 pelo barramento do rio Taboca e está localizado no município de Simão Dias. O material da barragem é de terra com um volume de $115.000 \mathrm{~m}^{3} \mathrm{e}$ foi construída para atender o abastecimento humano e práticas de irrigação [8, 24]. 
O clima na região segundo a classificação climática de Kopper denomina-se como BSh - Clima Semiárido quente. Caracterizado por escassez de chuvas e grande irregularidade em sua distribuição; baixa nebulosidade; forte insolação; índices elevados de evaporação, e temperaturas médias elevadas (por volta de $27^{\circ} \mathrm{C}$ ) e poucas chuvas com média de $800 \mathrm{~mm}$ por ano [22].

Os solos são do tipo Neossolos Litólicos Eutróficos e associações de Argissolos Vermelho Amarelo Eutrófico e solos Litólicos Eutróficos. O relevo caracteriza-se com declividade suave ondulado [22].

\section{-Reservatório do Jabiberi (JB)}

O reservatório do Jabiberi foi construído no período de 1985 a 1987 pelo barramento do rio Jabiberi e está situado no município de Tobias Barreto. Inunda uma área de 60 ha e tem um volume acumulada de $4.300 .000 \mathrm{~m}^{3}$. No seu entorno e na jusante do reservatório encontra-se a cidade de Tobias Barreto, e os povoados Jabiberi, Agrovila, Juazeiro, Geroma e o perímetro irrigado do Jabiberi. Os usos atuais da barragem são para abastecimento humano, piscicultura e abastecimento hidro agrícola [8].

Segundo a classificação climática de Koppen, o clima da região é do tipo As (clima quente, com temperatura do ar superior a $18{ }^{\circ} \mathrm{C}$ no mês mais frio e, no mês mais seco a precipitação pluvial é inferior a $60 \mathrm{~mm}$ e o verão é seco). A precipitação pluvial média anual é de $756,9 \mathrm{~mm}$; o mês de julho é o mais frio do ano, com média de $22,1^{\circ} \mathrm{C}$, e o mês de janeiro é o mais quente, com média de $25,7^{\circ} \mathrm{C}$; as temperaturas médias mínimas mensais estão compreendidas entre $18 \mathrm{e}$ $22{ }^{\circ} \mathrm{C}$ e as temperaturas médias máximas mensais estão entre 26 e $32^{\circ} \mathrm{C}$ [25].

Os solos prevalecentes nessa região são classificados como solos Halomórficos (SS2) e Litólicos (Re3, Re6 e Re2). O uso do solo na região de Tobias Barreto é composto pela maior parte pela pastagem, sendo o restante composto pela caatinga e cultivos agrícolas/solos expostos [24].

\section{-Reservatório da Amargosa (AG)}

O reservatório Amargosa foi construído em 1985, está localizado no município de Poço Verde e tem uma capacidade acumulada de $4.300 .000 \mathrm{~m}^{3}$ [8]. O município de Poço Verde na sua porção Leste possui um relevo ligeiramente montanhoso, representado pelas Serras da Caraíba, Poço Dantas e São José. Ainda nesta região do Estado localiza-se o ponto culminante, a Serra Negra, com $750 \mathrm{~m}$ de altitude, que junto às anteriores caracterizam o pediplano sergipano [3].

O clima predominante, segundo a classificação indicimétrica de Koppen, é do Tipo As' (Tropical Megatérmico de outono e inverno chuvosos), com transição para BSh (Quente Semiárido). Na região deste reservatório a precipitação média anual de $760 \mathrm{~mm}$, e período úmido entre os meses de abril e julho. A temperatura média do mês mais quente é de $26,1^{\circ} \mathrm{C}$, em janeiro, e do mês mais frio é de $21,4^{\circ} \mathrm{C}$, em julho (IBGE, 2001) apud Anholetto Junior (2013) [3].

O tipo de solo prevalecente na região é classificado como Planossolo (PLSe4), Halomórficos (SS2), Cambissolos (Ce2) e Litólicos (Re6) [24].

\subsection{Amostragem e análise química}

As amostras de água de superfície foram coletadas no período de junho/2013 a agosto de 2014 nos seguintes pontos: DM (645.503 E, 8.788.322 N UTM), TB (631.638 E, 8.810.394 N UTM), JB (619.205 E, 8.777.592 N UTM) e AG (588.495 E, 8.813.276 N UTM), compreendendo dois períodos com chuvas e um período seco.

As amostras foram acondicionadas, preservadas e transportadas para o laboratório de acordo com as recomendações do Standard Methods [4]. Estas foram analisadas no Instituto Tecnológico e de Pesquisas do Estado de Sergipe (ITPS), para determinação dos seguintes parâmetros: pH, turbidez (TUR), oxigênio dissolvido (OD), demanda bioquímica de oxigênio $\left(\mathrm{DBO}_{5}\right)$, sólidos totais dissolvidos $(\mathrm{STD})$, fósforo total $\left(\mathrm{P}_{\mathrm{t}}\right)$, nitrato $\left(\mathrm{NO}_{3}\right)$, coliformes termotolerantes $(\mathrm{COL}) \mathrm{e}$ temperatura da água ( $\mathrm{T}$, medida no campo). Todos os parâmetros foram determinados seguindo 
os protocolos do Standard Methods [4]. Na Tabela 2 estão descritos as variáveis analisadas e os métodos utilizados.

Tabela 2. Variáveis analisadas e métodos utilizados na avaliação

\begin{tabular}{|c|c|c|}
\hline Parâmetros & Técnica analítica & $\begin{array}{l}\text { Método de } \\
\text { referência }\end{array}$ \\
\hline$p H$ & Método potenciométrico & $* \mathrm{SM} 4500 \mathrm{H}^{+} \mathrm{B}$ \\
\hline Turbidez & Método nefelométrico & SM 2130 B \\
\hline Oxigênio dissolvido & Método de laboratório & SM $4500 \mathrm{O}$ \\
\hline $\mathrm{DBO}_{5}$ & Método respirométrico & SM $5210 \mathrm{D}$ \\
\hline Sólidos totais dissolvidos & Determinado pela medida da condutividade & SM $2510 \mathrm{~A}$ \\
\hline Temperatura da água & Medida em campo & - \\
\hline Fósforo total & ICP OES & - \\
\hline Coliformes termotolerantes & NMP Colilert 24 & SM 9223 B \\
\hline Nitrogênio - nitrato & Cromatografia iônica & US EPA 300.7 \\
\hline
\end{tabular}

Para garantia da qualidade analítica foram usados a calibração com padrões, a análise de reagentes em branco e determinações em replicatas. O laboratório também participa regularmente de programas de proficiência e tem acreditação para alguns parâmetros.

\subsection{Cálculo do Índice de Qualidade da Água (IQA)}

O método usado neste trabalho foi desenvolvido por Brown na década de 70 em parceria com a Fundação Sanitária Nacional dos Estados Unidos da América (NSF - National Sanitation Foundation) [6] e adaptado no Brasil pela Companhia de Tecnologia de Saneamento Ambiental de São Paulo (Cetesb), mantendo os mesmos pesos $(w=0,10)$ e curvas de qualidade estabelecidos pela NSF [2]. O cálculo do IQA é feito por meio do produtório ponderado dos nove parâmetros $(\mathrm{pH}$, turbidez, temperatura, oxigênio dissolvido, fósforo, nitrogênio, sólidos totais, coliformes termotolerantes, e demanda bioquímica de oxigênio), aplicando a Equação 1.

$$
I Q A=\prod_{i=1}^{n} q_{i}^{w_{i}}
$$

Sendo:

IQA = Índice de Qualidade das Águas, um número entre 0 e 100;

qi $=$ valor indicativo da qualidade da água para cada um parâmetro em função da concentração, obtido das curvas de qualidade para cada parâmetro;

wi = Valor atribuído a cada parâmetro de acordo com sua importância relativa no cálculo do IQA, é um número entre 0 e 1 .

\subsection{Análise de Componentes Principais - ACP}

$\mathrm{Na}$ análise de componentes principais a matriz dos dados foi constituída de 10 objetos e 9 variáveis. Os objetos foram as amostras dos quatro reservatórios nos dois períodos chuvosos e um período seco (Amargosa só no período chuvoso). As variáveis foram os parâmetros medidos: $\mathrm{pH}$, turbidez (TUR), oxigênio dissolvido (OD), demanda biológica de oxigênio ( $\left.\mathrm{DBO}_{5}\right)$, sólidos totais dissolvidos (STD), temperatura da água $(\mathrm{T})$, fósforo total $\left(\mathrm{P}_{\mathrm{t}}\right)$, coliformes termotolerantes $(\mathrm{COL})$ e nitrato (NO3). A análise foi realizada utilizando o programa PAST - Paleontological STatistics, Versão 3.04 (http://folk.uio.no/ohammer/past). Um valor- $\alpha$ de 0,05 foi adotado como o nível crítico para todos os testes estatísticos dando um nível de confiança de $95 \%$. 


\section{RESULTADOS E DISCUSSÃO}

Na Tabela 3 são apresentados os valores dos parâmetros de qualidade da água, para as amostras coletadas nos três períodos de amostragem e na Tabela 4 encontram-se os valores calculados do qi para cada parâmetro, o IQA e a classificação da água dos reservatórios com base no valor do IQA.

Tabela 3. Valores brutos dos parâmetros coletados em campo. ${ }^{1} \mathrm{PC}$ (período chuvoso), PS (período seco)

\begin{tabular}{ccccccccccc}
\hline \multirow{2}{*}{ Período $^{1}$} & \multirow{2}{*}{ Reserv. } & \multicolumn{10}{c}{ Valores } \\
\cline { 3 - 11 } & & pH & TUR & OD & DBO5 & STD & NO $_{3}$ & $\mathbf{P}_{\mathbf{t}}$ & COL & T \\
\hline PC/2013 & AG & 7,8 & 41,4 & 4,65 & 19,1 & 2881 & 0,52 & 0,38 & 170 & 24 \\
PC/2013 & JB & 7,2 & 26,8 & 5,46 & 18,6 & 113,9 & 0,21 & 0,01 & 11 & 25 \\
PC/2014 & JB & 7,0 & 4,8 & 5,33 & 8,8 & 110,2 & 1,21 & 0,55 & 33 & 24 \\
PS/2013 & JB & 7,7 & 21,1 & 5,73 & 18,0 & 165,1 & 0,17 & 0,06 & 2 & 26 \\
PC/2013 & TB & 7,5 & 11,8 & 6,03 & 10,4 & 905,8 & 0,21 & 0,05 & 61 & 23 \\
PC/2014 & TB & 8,1 & 0,7 & 6,97 & 11,6 & 1397 & 0,01 & 0,51 & 350 & 26 \\
PS/2013 & TB & 8,0 & 5,4 & 3,28 & 18,5 & 1047 & 0,94 & 0,01 & 130 & 30 \\
PC/2013 & DM & 7,1 & 49,2 & 5,14 & 12,0 & 300,7 & 0,29 & 0,83 & 11 & 25 \\
PC/2014 & DM & 8,7 & 24,6 & 10,71 & 4,9 & 667,0 & 0,01 & 0,51 & 700 & 31 \\
PS/2013 & DM & 9,5 & 10,4 & 10,99 & 4,14 & 565,2 & 0,01 & 0,01 & 12 & 30 \\
\hline
\end{tabular}

Tabela 4: Valores do IQA, qi para cada subindices (parâmetros) e classificação da água dos reservatórios

\begin{tabular}{|c|c|c|c|c|c|c|c|c|c|c|c|c|}
\hline \multirow{2}{*}{ Período $^{1}$} & \multirow{2}{*}{ Reserv. } & \multicolumn{9}{|c|}{ Qi } & \multirow{2}{*}{ IQA } & \multirow{2}{*}{ Clas. $^{2}$} \\
\hline & & OD & $\mathbf{T}$ & $\mathrm{DBO}_{5}$ & $\mathrm{NO}_{3}$ & $\mathbf{P}_{\mathbf{t}}$ & STD & TUR & pH & COL & & \\
\hline PC/2013 & $\mathrm{AG}$ & 33 & 94 & 12 & 83 & 37 & 32 & 43 & 89 & 35 & 43 & ACEIT \\
\hline PC/2013 & JB & 42 & 94 & 13 & 93 & 96 & 85 & 55 & 91 & 65 & 60 & BOA \\
\hline PC/2014 & JB & 40 & 94 & 75 & 65 & 30 & 85 & 88 & 88 & 52 & 61 & BOA \\
\hline PS/2013 & $\mathrm{JB}$ & 46 & 94 & 14 & 94 & 83 & 79 & 61 & 90 & 90 & 64 & BOA \\
\hline PC/2013 & TB & 51 & 94 & 73 & 93 & 86 & 32 & 74 & 92 & 45 & 66 & BOA \\
\hline PC/2014 & $\mathrm{TB}$ & 65 & 94 & 71 & 100 & 31 & 32 & 98 & 83 & 29 & 59 & BOA \\
\hline PS/2013 & $\mathrm{TB}$ & 21 & 94 & 13 & 71 & 96 & 32 & 87 & 85 & 38 & 46 & ACEIT \\
\hline PC/2013 & DM & 38 & 94 & 71 & 90 & 20 & 60 & 38 & 90 & 65 & 56 & BOA \\
\hline PC/2014 & $\mathrm{DM}$ & 98 & 94 & 55 & 100 & 31 & 32 & 57 & 65 & 23 & 55 & BOA \\
\hline PS/2013 & DM & 100 & 94 & 60 & 100 & 96 & 32 & 77 & 35 & 64 & 69 & BOA \\
\hline
\end{tabular}

${ }^{I} P C$ (período chuvoso), $P S$ (período seco); ${ }^{2}$ Clas. (Classificação): Ótima $(80 \leq I Q A<100)$, Boa $(52 \leq$ $I Q A<80)$, Aceit (aceitável) $(37 \leq I Q A<52)$, Ruim $(20 \leq I Q A<37)$, Péssima $(I Q A<20)$.

Conforme a Tabela 4, com exceção dos reservatórios AG e TB no período seco de 2013, em todos os outros reservatórios a água foi classificada como "boa". É possível observar também na Tabela 4, que apesar da classificação "boa", alguns subíndices tiveram valores baixos e que inclusive para um mesmo reservatório, os subíndices de valores baixos mudaram de uma campanha para outra. Essa é considerada uma fraqueza inerente à medida do IQA, que apesar da existência de valores prejudiciais de certos parâmetros, leva a uma classificação "boa", pelo fato de encobrir valores baixos dos subíndices. Por isso a avaliação de cada subíndice, associado ao valor do IQA é fundamental, se se pretende usar o IQA como uma ferramenta de gerenciamento de decisões e intervenções [12]. Os subíndices têm como objetivo expressar valores dos parâmetros em unidades comuns de qualidade da água tipicamente baseadas em uma escala de zero (pior) a 100 (melhor). Para efeito da avaliação dos subíndices será utilizada a seguinte classificação: valores menores que 25 são considerados muito ruim, valores entre 25 a 75 são considerados médio, e valores acima de 75 são considerados de boa qualidade. 
Para o reservatório AG no período chuvoso, o subíndice da $\mathrm{DBO}_{5}$ (qi=12) foi a variável de maior impacto, levando a classificação para "aceitável"; seguida pelo STD (qi=32), OD (qi=33) e COL (qi=35).

No reservatório JB, os subíndices com menores valores foram a $\mathrm{DBO}_{5}$ (qi=13) e OD (qi=42) no período chuvoso de 2013 (PC/2013); $\mathrm{P}_{\mathrm{t}}(\mathrm{qi}=30)$ e $\mathrm{OD}$ (qi=40) no período chuvoso de 2014 (PC/2014) e $\mathrm{DBO}_{5}$ (qi=14) e OD (qi=46) no período seco de 2013 (PS/2013). Ao comparar os valores dos subíndices para as três campanhas, observa-se em geral, um comportamento semelhante entre os valores obtidos nas campanhas PC/2013 e PS/2013, e uma diferença em $\mathrm{PC} / 2014$, principalmente para a $\mathrm{DBO}_{5}(75)$ e $\mathrm{P}_{\mathrm{t}}(30)$. Desse modo, o impacto mais significativo $(\mathrm{qi}<25)$ no reservatório $\mathrm{JB}$ está relacionado às maiores concentrações de $\mathrm{DBO}_{5}$ nos períodos $\mathrm{PC} / 2013$ e PS/2013.

Para o reservatório $\mathrm{TB}$, os menores valores dos subíndices foram obtidos para os parâmetros STD (qi=32) e COL (qi=45) no período chuvoso PC/2013, enquanto no período chuvoso PC/2014 os menores valores foram registrados para o $\mathrm{P}_{\mathrm{t}}(\mathrm{qi}=31)$ e STD (qi=32). Já no período seco de 2013 (PS/2013) observou-se valores baixos para $\mathrm{DBO}_{5}$ (qi=13), OD (qi=21), STD (qi=32) e COL (qi=38). Comparando as três campanhas, o período seco (PS/2013) se destaca dos demais por apresentar valores dos subíndices menores que 25 para os parâmetros $\mathrm{OD}$ e $\mathrm{DBO}_{5}$, levando a classificação da água do reservatório para "aceitável".

No reservatório $\mathrm{DM}$ os baixos valores dos subindices foram para o $\mathrm{P}_{\mathrm{t}}(\mathrm{qi}=20)$, turbidez ( $\mathrm{qi}=38$ ) e OD (qi=38), no PC/2013; para o PC/2014 os subíndices de maior impacto foram $\mathrm{P}_{\mathrm{t}}$ (qi=31), STD (qi=32) e COL (qi=23); enquanto no PS/2013 foram registados valores baixos dos subindices para STD (qi=32) e $\mathrm{pH}(\mathrm{qi}=35)$. Comparando as três campanhas verifica-se que o maior IQA ocorreu no período seco (PS/2013) e nos dois períodos chuvosos (PC/2013, PC/2014) os valores do IQA foram semelhantes e menores do que o IQA do período seco. Os dois principais subíndices que contribuíram para redução do IQA no período chuvoso foram $\mathrm{P}_{\mathrm{t}}(\mathrm{qi}=20)$ no PC/2013 e COL (qi=23) no PC/2014. Portanto os impactos mais significativos (subíndice <25) na água do reservatório DM estão associados aos parâmetros $\mathrm{P}_{\mathrm{t}} \mathrm{e} C \mathrm{COL}$.

O IQA usado nesse estudo tem uma limitação em relação aos STD, pois atribui para valores de STD acima de $500 \mathrm{mg} \mathrm{L}^{-1}$ um valor de qi constante e igual a 32. Na Tabela 3 observa-se que valores de STD acima de $500 \mathrm{mg} \mathrm{L}^{-1}$ ocorreram para amostra de água dos reservatórios nas seguintes campanhas: AG (PC/2013), TB (PC/2013 e PS/2013) e DM (PC/2014 e PS/2013). Na Tabela 4 verifica-se que o qi do parâmetro STD para todas essas amostras foi igual a 32, independentemente do valor absoluto dos STD, ou seja, apesar dos diferentes valores do STD, não é feita uma diferenciação da qualidade desse subíndice (qi), para efeito da medida do IQA, quando os valores ultrapassam $500 \mathrm{mg} \mathrm{L}^{-1}$.

Para avaliar o impacto dos STD na classificação das amostras, foi aplicada a Análise de Componentes Principais (ACP) ao conjunto dos dados da qualidade da água dos reservatórios (Tabela 3), com o objetivo de perceber um possível agrupamento das amostras e a contribuição dos STD na formação dos grupos.

A ACP reduziu o número de variáveis iniciais (9) para apenas duas componentes principais (CP1 e CP2), guardando $100 \%$ das informações contidas no conjunto dos dados da qualidade da água dos reservatórios. A CP1 foi responsável por $94 \%$ da variância explicada e está associada aos STD (peso=0,997), enquanto a CP2 está associada aos COL e respondeu por 6,0\% da variância total (Tabela 5).

A Figura 2 mostra a localização das amostras no plano de coordenadas formado pelas componentes CP1 e CP2. A ACP separou as amostras de água dos reservatórios em quatro grupos: Grupo I, formado pelas amostras dos reservatórios JB (PC/2013, PC/2014, PS/2013), TB (PC/2013, PS/2013) e DM (PC/2013, PS/2013); Grupo II, composto por TB (PC/2014); Grupo III, contém a amostra do reservatório AG (PC/2013) e o Grupo IV, a amostra DM (PC/2014). Os Grupos I e III estão separados em função dos valores dos STD, que crescem no sentido do Grupo I para o Grupo III. As amostras DM (PC/2014) e TB (PC/2014) se separaram do Grupo I, por apresentarem valores de COL significativamente maiores do que os valores dos demais componentes do Grupo I. 
Tabela 5: Pesos dos parâmetros experimentais nas Componentes Principais

\begin{tabular}{lcc}
\hline \multirow{2}{*}{ Parâmetros da qualidade da água } & \multicolumn{2}{c}{ Componentes Principais } \\
\cline { 2 - 3 } pH & $\mathrm{CP} 1$ & $\mathrm{CP} 2$ \\
Turbidez (TUR) & 0,000 & 0,001 \\
Temperatura (T) & 0,003 & $-0,009$ \\
Oxigênio dissolvido (OD) & 0,000 & 0,007 \\
Demanda biológica de oxigênio (DBO 5$)$ & 0,000 & 0,007 \\
Sólidos totais dissolvidos (STD) & 0,002 & $-0,012$ \\
Nitrato $\left(\mathrm{NO}_{3}\right)$ & 0,997 & $-0,074$ \\
Fósforo total $\left(\mathrm{P}_{\mathrm{t}}\right)$ & 0,005 & 0,000 \\
Coliformes termotolerantes (COL) & 0,251 & 0,000 \\
\hline
\end{tabular}

Dentro de cada Grupo estão as amostras com Qualidade da Água estatisticamente similares. Isso significa que a água da amostra TB (PC/2014) deve ter uma classificação diferente de TB (PC/2013) e similar a TB (PS/2013) e contribuiu para essa nova classificação os valores do STD. Enquanto AG (PC/2013) deve ter uma classificação diferente das demais amostras, devido ao elevado valor dos STD. Já a amostra DM (PC/2014) difere das amostras DM (PC/2013) e DM (PS/2013) pela maior concentração de COL, e, portanto, deve ter uma classificação diferenciada em relação às amostras DM (PC/2013) e DM (PS/2013). Por conta disso, na Tabela 6 comparase a classificação obtida pelo IQA com a sugerida a partir do agrupamento indicado pela ACP, e propõem-se uma classificação final para Qualidade da Água dos reservatórios, levando em consideração a contribuição da ACP para resolver a limitação do IQA, em relação aos valores do qi para o subíndice STD.

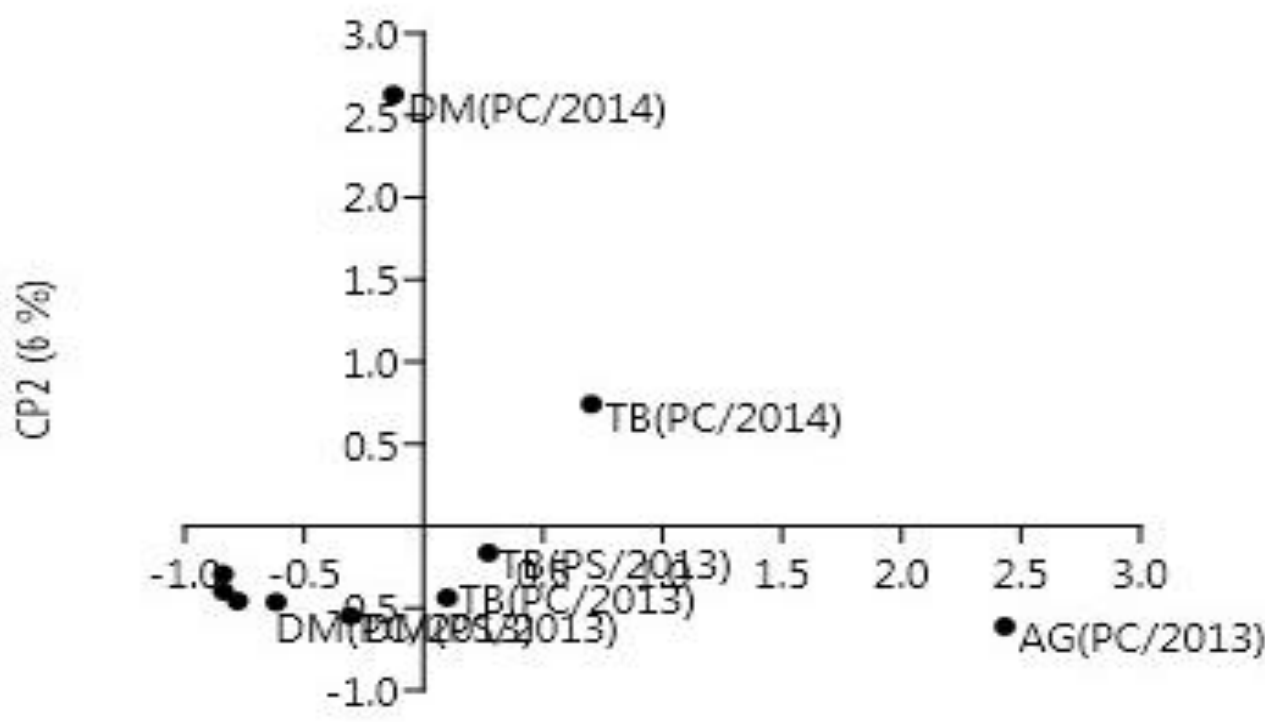

CP1 (94\%)

Figura 2. Agrupamento das amostras dos reservatórios no plano formado pelas componentes CP1 e CP2. 
Tabela 6: Comparação entre a classificação obtida pelo IQA e a sugerida pela ACP, e proposta de classificação da qualidade da água dos reservatórios.

\begin{tabular}{|c|c|c|c|c|}
\hline \multirow{2}{*}{ Período } & \multirow{2}{*}{ Reservatório } & \multicolumn{3}{|c|}{ Classificação } \\
\hline & & $\begin{array}{l}\text { IQA } \\
\end{array}$ & $\mathbf{A C P}$ & $\begin{array}{l}\text { PROPOSTA } \\
\end{array}$ \\
\hline $\mathrm{PC} / 2013$ & AG & ACEITÁVEL & RUIM & RUIM \\
\hline PC/2013 & $\mathrm{JB}$ & BOA & $\mathrm{BOA}$ & BOA \\
\hline PC/2014 & JB & BOA & BOA & $\mathrm{BOA}$ \\
\hline PS/2013 & $\mathrm{JB}$ & $\mathrm{BOA}$ & BOA & BOA \\
\hline PC/2013 & ТВ & BOA & BOA & BOA \\
\hline PC/2014 & TB & $\mathrm{BOA}$ & ACEITÁVEL & ACEITÁVEL \\
\hline PS/2013 & ТВ & ACEITÁVEL & BOA & ACEITÁVEL \\
\hline PC/2013 & DM & $\mathrm{BOA}$ & BOA & BOA \\
\hline PC/2014 & DM & BOA & ACEITÁVEL & ACEITÁVEL \\
\hline PS/2013 & DM & $\mathrm{BOA}$ & BOA & $\mathrm{BOA}$ \\
\hline
\end{tabular}

\section{CONCLUSÃO}

O Índice de Qualidade da Água dos reservatórios localizados nas bacias hidrográficas dos rios Piauí - Real foi calculado usando o IQA multiplicativo, desenvolvido pela NSF (National Sanitation Foundation) e adaptado no Brasil pela Companhia de Tecnologia de Saneamento Ambiental de São Paulo (CETESB). A Análise de Componentes Principais (ACP) foi utilizada com ferramenta auxiliar, para resolver a limitação do IQA, em relação aos valores de sólidos totais dissolvidos (STD) superiores a $500 \mathrm{mg} \mathrm{L}^{-1}$.

No período estudado (junho/2013 - agosto/2014) a água do reservatório Amargosa foi classificada como "Ruim" e a do reservatório Jabiberi como "Boa". Para os reservatórios Taboca e Dionísio Machado a qualidade da água variou de "Aceitável" para "Boa" em função do período de amostragem.

Os parâmetros que mais impactaram a qualidade da água foram $\mathrm{DBO}_{5}$, oxigênio dissolvido, coliformes termotolerantes e sólidos totais dissolvidos, e devem estar associados, principalmente, ao aporte de despejos sanitários e a processos de salinização.

\section{AGRADECIMENTOS}

Esse trabalho contou com o apoio do projeto "Monitoramento de Mananciais Superficiais e Reservatórios de Sergipe", convênio 001/2012 entre a Secretária de Estado do Meio Ambiente e Recursos Hídricos do Estado de Sergipe (SEMARH) e o Instituto Tecnológico e de Pesquisas de Sergipe (ITPS). Por isso, apresentamos os nossos agradecimentos à SEMARH e ao ITPS.

\section{REFERÊNCIAS BIBLIOGRÁFICAS}

1. Abbasi T, Abbasi SA. Water quality indices. Amsterdam: Elsevier; 2012. 384 p.

2. ANA. Agência Nacional de Águas. Panorama da qualidade das águas superficiais no Brasil; 2012. 264 p. [Acesso em 3 de março de 2017]. Disponível em: http://arquivos.ana.gov.br/institucional/sge/CEDOC/Catalogo/2012/PanoramaAguasSuperficiaisPortu gues.pdf

3. Anholetto Junior CR. Dendroecologia e composição isotópica $\left(\delta^{13} \mathrm{C}\right)$ dos anéis de crescimento de árvores de Cedrela odorata, Meliaceae, na Caatinga e Mata Atlântica do Estado de Sergipe, Brasil [dissertação]. Universidade de São Paulo. Escola Superior de Agricultura "Luiz de Queiroz"; 2013. 91 p.

4. APHA. Standard Methods for Examination of Water and Wastewater. American Public Health Association, Washington, DC; 2012. 1496 p. 
5. Bastos RKX. Impactos da construção de centrais hidrelétricas relacionados com a água: pressupostos para a avaliação e proposição de medidas mitigadoras. In: Simpósio brasileiro de pequenas e médias centrais hidrelétricas, 1; 1998, Poços de Caldas. Anais. São Paulo: CMGB. 1998. p. 63-75.

6. Brown RM, McClelland NI, Deininger RA, Tozer RG. A water quality index- do we dare? Water Sewage Works. 1970;117:339-343.

7. Carpenter SR, Stanley EH, Zanden MJV. State of the World's Freshwater Ecosystems: Physical, Chemical, and Biological Changes. Annual Review Environment Resources. 2011;36:75-99, doi:org/10.1146/annurev-environ-021810-094524.

8. COHIDRO - Companhia de Desenvolvimento e Irrigação. [Acesso em 3 de março de 2017]. Disponível em: http://www.cohidro.se.gov.br/modules/tinyd0/index.php?id=14

9. Conama, Resolução. 357, de 17 de março de 2005. Conselho Nacional do Meio Ambiente-CONAMA, v. 357, 2005.

10. Ferreira KCD, Lopes FB, De Andrade EM, Meireles ACM, Da Silva GS. Adaptação do índice de qualidade de água da National Sanitation Foundation ao semiárido brasileiro. Revista Ciência Agronômica. 2015;46(2):277-286, doi:10.5935/1806-6690.20150007.

11. Ferreira NC, Bonetti C, Seiffert WQ. Hydrological and water quality indices as management tools in marine shrimp culture. Aquaculture. 2011;318(3):425-433, doi:org/10.1016/j.aquaculture.2011.05.045.

12. Gitau MW, Chen J, Ma Z. Water quality indices as tools for decision making and management. Water Resources Management. 2016;30(8):2591-2610, doi:10.1007/s11269-016-1311-0.

13. Gupta AK, Gupta SK, Patil RS. A comparison of water quality indices for coastal water. Journal of Environmental Science and Health, Part A. 2003;38(11):2711-2725, doi:10.1081/ESE-120024458.

14. Hurley T, Sadiq R, Mazumder A. Adaptation and evaluation of the Canadian Council of Ministers of the Environment Water Quality Index (CCME WQI) for use as an effective tool to characterize drinking source water quality. Water Research. 2012;46(11):3544-3552, doi:10.1016/j.watres.2012.03.061.

15. Lima RS, Fonseca LC, Macedo LCB, Passos EA, Alves JPH. Variações na qualidade da água superficial dos reservatórios das bacias dos rios PIAUÍ-REAL/Sergipe. In: XII Simpósio de Recursos Hídricos do Nordeste; 2014 Nov 04 - 07; Natal/RN.

16. Lima RS. Qualidade da água dos reservatórios situados na bacia hidrográfica dos rios Piauí - Real: uma avaliação com base em técnicas estatísticas multivariadas e razões iônicas [dissertação]. São Cristóvão (SE): Universidade Federal de Sergipe; 2015. 101 p.

17. Lima, RS, Fonseca LC, Macedo LCB, Alves JPH. Qualidade da água dos reservatórios situados na bacia hidrográfica dos rios Piauí-Real: uma avaliação com base em técnicas estatísticas multivariadas e razões iônicas. In: XXI Simpósio Brasileiro de Recursos Hídricos; 2015 Nov 17 - 22; Brasília (DF).

18. Liu Y, Chen W, Li D, Huang Z, Shen Y, Liu Y. Cyanobacteria-/cyanotoxin-contaminations and eutrophication status before Wuxi drinking water crisis in Lake Taihu, China. Journal of Environmental Sciences. 2011;23(4):575-581, doi:org/10.1016/S1001-0742(10)60450-0.

19. Lumb A, Sharma TC, Bibeault JF. A review of genesis and evolution of water quality index (WQI) and some future directions. Water Quality, Exposure and Health. 2011;3(1):11-24, doi:10.1007/s12403011-0040-0.

20. Poonam T, Tanushree B, Sukalyan C. Water quality indices-important tools for water quality assessment: a review. International Journal of Advances in Chemistry. 2013;1(1):15-28.

21. Sánchez E, Colmenarejo MF, Vicente J, Rubio A, Garcia MG, Travieso L, Borja R. Use of the water quality index and dissolved oxygen deficit as simple indicators of watersheds pollution. Ecological Indicators. 2007;7(2):315-328, doi:10.1016/j.ecolind.2006.02.005.

22. Santos C, Pedrotti A, Ramos OF, Rodrigues SAS. Aspectos da sustentabilidade no cultivo do milho no estado de Sergipe. In: XXI Encontro Nacional de Geografia Agrária; 2012 Out 15 - 19; Uberlândia (MG).

23. Santos CM. Ruralidades agrícolas e não Agrícolas em Lagarto-SE [Tese]. São Cristóvão (SE): Universidade Federal de Sergipe; 280 p. 2009.

24. Sergipe. Secretaria de Estado do Planejamento e da Ciência e Tecnologia - Superintendência de Recursos Hídricos. Sergipe: Atlas Digital Sobre Recursos Hídricos. CD-ROM; 2012.

25. Sousa IF, Silva VPR, Sabino FG, Netto AO, Silva BKN, Azevedo PV. Evapotranspiração de referência nos perímetros irrigados do Estado de Sergipe. Revista Brasileira de Engenharia Agrícola e Ambiental, Campina Grande. 2010;14(6):633-644, doi:org/10.1590/S1415-43662010000600010.

26. Tundisi JG. Represas artificiais: perspectivas para o controle e manejo da qualidade da água para usos múltiplos. In: Anais VI Simpósio Brasileiro de Hidrologia e Recursos Hídricos; 1985; São Paulo (SP), p. 38-59. 